\title{
Exact Sub-Systems and their Link to Modelling
}

\author{
Janina Dierkes* Dirk Langemann* \\ * Institute of Computational Mathematics, Technische Universität \\ Braunschweig, Germany (e-mail: j.dierkes@tu-bs.de).
}

Keywords: mathematical modelling, model families, sub-models, approximation

\section{INTRODUCTION}

The approximation of a high-dimensional, discrete or continuous, quantified or even non-quantified system by a lower-dimensional well-quantified system occurs in multiscale numerics, cf. Ames (1969), in lower-dimensional representations and in modelling, cf. Murray (2008). In particular in modelling, the small system is interpreted as a model for the larger system, oftentimes a real-world problem. Here, we discuss basic ideas by hands of finite dimensional specifications.

We regard a system of ordinary differential equations

$$
\dot{X}=F(X), X(0)=X^{\text {ini }} \in \Omega \subseteq \mathbb{R}^{N}
$$

in $X=X(t) \in \mathbb{R}^{N}$ with the initial value $X^{\text {ini }}$. In general, the $N$ equations in (1) are coupled. We aim to approximate this system by a lower dimensional system in $Y \in \mathbb{R}^{M}$, $M<N$ and sometimes formally $M \leq N$, named

$$
\dot{Y}=G(Y), Y(0)=Y^{\text {ini }} \in \Theta \subseteq \mathbb{R}^{M}
$$

with the initial value $Y^{\text {ini }}$. One essential question is wether $Y(t) \approx \varphi(X(t))$ with a link map $\varphi: \mathbb{R}^{N} \rightarrow \mathbb{R}^{M}$ is an appropriate approximation for certain $X^{\text {ini }} \in \Omega, Y^{\text {ini }} \in \Theta$ and $t \in[0, T]$.

Definition 1. We call the system $\dot{Y}=G(Y)$ an exact subsystem of $\dot{X}=F(X)$, if $Y(t)=\varphi(X(t))$ for all $t \in[0, T]$ results from $Y^{\text {ini }}=\varphi\left(X^{\text {ini }}\right)$ for all $X^{\text {ini }} \in \Omega$.

Remark 2. Equation (2) needs not to be a sub-system in the sense that it is a part of (1). Here, we understand an exact sub-system as the existence of an exact link map $\varphi$.

Example 3. Let us regard the harmonic oscillator with $F: \mathbb{R}^{2} \rightarrow \mathbb{R}^{2}$ and $\dot{X}=F(X)=\left(X_{2},-X_{1}\right)^{\mathrm{T}}$. Even for this comparably simple system, we find surprisingly many exact sub-systems. By transition to complex variables, we find the two sub-systems $\dot{Y}= \pm \mathrm{i} Y=G(Y)$ with $\varphi: \mathbb{R}^{2} \rightarrow \mathbb{C}$ by $\varphi(X)=\frac{1}{2}(1, \mp \mathrm{i})^{\mathrm{T}} \cdot X=Y$, which are independent from each other. By

$$
\varphi: X \mapsto c\left(X_{1}^{2}+X_{2}^{2}\right)=Y
$$

with $\dot{Y}=G(Y)=0$, we generate a sub-system containing the conservation of energy. In addition,

$$
\varphi: X \mapsto t=Y=\arctan \frac{-X_{2}}{X_{1}}
$$

and $G=1$ pose an unexpected exact sub-system of the harmonic oscillator as long as $0 \leq t<2 \pi$ holds.

These four exact sub-systems are part of the model family of all models of the harmonic oscillator.

\section{TERMS, DEFINITIONS AND PROPERTIES}

A particularly simple sub-system is induced by a selection of components of $X$. Such a selection is a projection

$$
\pi_{J}: X=\left(X_{1}, \ldots, X_{N}\right)^{T} \mapsto\left(X_{j_{1}}, \ldots, X_{j_{k}}\right)^{T}=Y
$$

with a set of indices $J=\left\{j_{1}, \ldots, j_{k}\right\}$. The projection $\pi_{J}: \mathbb{R}^{N} \rightarrow \mathbb{R}^{M}$ with $M=|J|$ defines the link $\varphi=\pi_{J}$ between the system in $X \in \mathbb{R}^{N}$ and a sub-system in $Y \in \mathbb{R}^{M}$.

\subsection{Reducibility, separability, adjacency structure}

Definition 4. A system (1) is called reducible, if there is a set $J \subset I=\{1, \ldots, N\}, J \neq I$, forming an exact subsystem with $\varphi=\pi_{J}$ and $G\left(\pi_{J} X\right)=\pi_{J} F(X)$. A system (1) is called separable, if $\varphi=\pi_{I \backslash J}$ forms an exact sub-system, too.

Reducibility and separability are recognized by the adjacency structure of $\nabla F$. A system (1) is reducible if $\nabla F \in \mathbb{R}^{N \times N}$ is an identically reducible matrix for all $X \in \Omega$. It is separable if $\nabla F$ has block structure.

Furthermore, $\nabla F$ displays the causal dependency structure of the system (1), i.e. $X_{i}$ causally depends on $X_{j}$, if the entry $(\nabla F)_{j i}$ in the $j$-th row and $i$-th column of the matrix $\nabla F$ is non-vanishing for some $X \in \Omega$.

\subsection{Coordinates and concepts}

The terms of reducibility and separability depend on coordinates. In order to achieve a causal structure, which is as simple as possible, coordinates are sought, in which the system is reducible or even separable. This search is formalised by a transformation $\mathcal{T}: \mathbb{R}^{N} \rightarrow \mathbb{R}^{N}$ with a bijective diffeomorphism $\mathcal{T}: X \mapsto X^{\prime}$. The transformed system in $X^{\prime} \in \mathbb{R}^{N}$ is

$$
\dot{X}^{\prime}=\left[\nabla \mathcal{T}^{-1} X^{\prime}\right] \cdot F\left(\mathcal{T}^{-1} X^{\prime}\right)=\tilde{F}\left(X^{\prime}\right) .
$$

If there is a transformation so that the system (4) is reducible or separable, we call the system (1) potentially reducible or separable, respectively.

\subsection{Linear systems}

Applied to linear systems $\dot{X}=A X=F(X)$ with $A \in$ $\mathbb{R}^{N \times N}$, we look for a linear sub-system $\dot{Y}=B Y=G(Y)$ with $B \in \mathbb{R}^{M \times M}$ and initial values as mentioned above. 
A spectral decomposition induces a transformation $\mathcal{T}$ : $R^{N} \rightarrow \mathbb{R}^{N}$ given by

$$
X^{\prime}=\mathcal{T} X:=V^{-1} X
$$

where $V \in \mathbb{C}^{N \times N}$ contains the eigenvectors of $A$ with $A V=V \Lambda$. Then, (4) is $\dot{X}^{\prime}=\Lambda X^{\prime}$, where $\Lambda \in \mathbb{C}^{N \times N}$ is the Jordan matrix of eigenvalues.

In case of a non-diagonalisable matrix $A$, the transformed system separates into Jordan blocks. Each Jordan block represents an exact sub-system, not further separable. Let us regard an upper tridiagonal $r \times r$-Jordan block between the rows $m-r+1$ and $m$ in $\Lambda$. Then, all projections

$$
\varphi=\pi_{J} \mathcal{T}: X \mapsto\left(X_{m-j}^{\prime}, \ldots, X_{m}^{\prime}\right)^{\mathrm{T}}=Y
$$

with $0 \leq j \leq r-1$ induce exact sub-systems. An amount of $k$ Jordan blocks is equivalent to $k$ independent subsystems. In particular the system $\dot{X}^{\prime}=\Lambda X^{\prime}$ is separable if $\Lambda$ contains more than one Jordan block, because they depict sub-systems that can be described independently from each other.

In case of a diagonalisable $A$, the matrix $\Lambda$ is diagonal. Hence, the system $\dot{X}^{\prime}=\Lambda X^{\prime}$ is completely separable. All projections $\varphi=\pi_{I} \mathcal{T}: X \mapsto Y$ with any set of indices $I=\left\{i_{1}, \ldots, i_{m}\right\} \subseteq\{1, \ldots, N\}$ induce exact subsystems. In contrast to the non-diagonalisable Jordan case, any selection of components is admissible, and the system $\dot{X}^{\prime}=\Lambda X^{\prime}$ has a very simple causal structure.

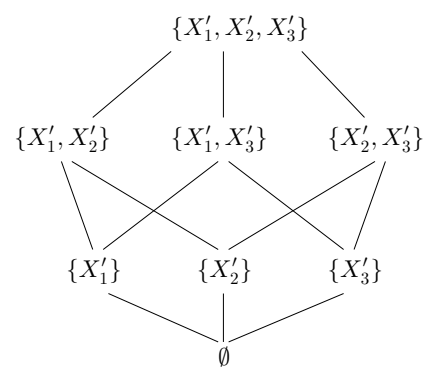

(a) diagonal

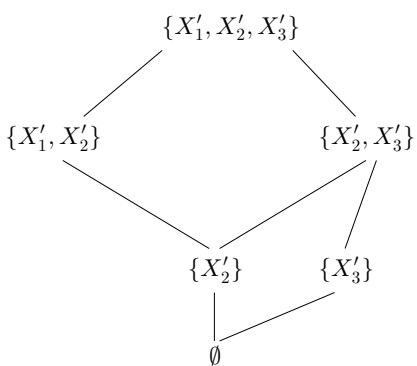

(b) Jordan
Fig. 1. Families of exact linear sub-systems for $N=3$. Left: diagonalisable $A$, full family. Right: Two Jordan blocks, one with $r=2, m=2$, and one with $r=1$, $m=3$ with separation in $\left\{X_{1}^{\prime}, X_{2}^{\prime}\right\}$ and $\left\{X_{3}^{\prime}\right\}$. Thus $\left\{X_{1}^{\prime}\right\}$ is not a stand-alone exact sub-system.

\subsection{One-dimensional situation}

In the one-dimensional case with $N=M=1$, we have scalar quantities $X$ and $Y$. Consequently, we find $F, G: \mathbb{R} \rightarrow \mathbb{R}$ and the link $\varphi: \mathbb{R} \rightarrow \mathbb{R}$. So the systems (1) and (2) are one-dimensional autonomous ordinary differential equations. Solutions of such equations always are monotonous. Furthermore, differentiable and monotonous functions $\mathbb{R} \rightarrow \mathbb{R}$ can be transformed into each other by a non-linear scaling of the domain and co-domain. Consequently, every one-dimensional system is an exact sub-system of every one-dimensional differential equation according to Def. 1. The impact of this surprising observation in modelling will be analysed in further research.

\subsection{Noether's theorem and conserved quantities}

Noether's theorem explains the connection between the existence of conserved quantities and the symmetry of a sys- tem under transformation of variables, cf. Boccaletti and Pucacco (2001). The invariance under spacial translations leads to momentum conservation, while the conservation of energy results from the invariance under time translation, as in Example 3. In the formalism introduced here, a conserved quantity $Y$ of (1), like in Noether's theorem, is described by an exact sub-system in (2) with $M=1$ and $G=0$ what implies $Y \in \mathbb{R}$ with $\dot{Y}=0$.

\section{THE MODELLING POINT OF VIEW}

We have introduced a conceptual framework that describes the approximation of $\dot{X}=F(X)$ by lower-dimensional sub-systems $\dot{Y}=G(Y)$. The modelling point of view takes the system (1) as a real-world system, we want to describe by a model (2). In the context of modelling, we interpret the transformation $\mathcal{T}: X \mapsto X^{\prime}$ as a choice of terms or concepts, that induce a well-arranged causal structure that is as simple as possible. While describing physical or biological systems, scientists always search for simple causal dependencies and simple descriptions, cf. Machamer and Silberstein (2002). The projection $\pi_{J}$ induced by (3) represents the selection of components to consider in the model. The presented conceptual framework fits into the process of modelling and forms an approach for its formalisation.

\section{OUTLOOK}

An intuitive approach to modelling starts with an identification of basic mechanisms. In order to model a system (1), we assume separated and independent mechanisms. These mechanisms are represented by functions $F_{i}: \mathbb{R}^{N} \rightarrow \mathbb{R}^{N}$ in $F=\alpha_{1} F_{1}+\ldots+\alpha_{k} F_{k}$ with fixed $\alpha_{1}, \ldots, \alpha_{k} \in \mathbb{R}$. This leads to the system equation

$$
\dot{X}=\alpha_{1} F_{1}(X)+\ldots+\alpha_{k} F_{k}(X) .
$$

It is determined, which mechanism leads to which change of the system (5). One question to analyse is, how the terms of reducibility and separability fit to the separation of mechanisms and the resulting linear combination of different $F_{i}$.

Another point of view might interpret the $\alpha_{i}$ as parameters, defining different models to identify. The resulting parameter identification is a model identification in order to find a best model of a real-world problem and thus a best approximation of the system (1).

\section{REFERENCES}

Ames, W.F. (1969). Numerical Methods for Partial Differential Equations. Nelson, London.

Boccaletti, D. and Pucacco, G. (2001). Theory of Orbits: Integrable Systems and Non-Perturbative Methods. Springer, Berlin.

Machamer, P. and Silberstein, M. (eds.) (2002). The Blackwell Guide to the Philosophy of Science. Blackwell Publishers, Oxford.

Murray, J. (2008). Mathematical Biology. Springer, New York. 